\title{
Role of ATP in Extracellular Vesicle Biogenesis and Dynamics
}

\author{
Marta Lombardi ${ }^{1}$, Martina Gabrielli ${ }^{1}$, Elena Adinolfi ${ }^{2}$ and Claudia Verderio ${ }^{1 *}$ \\ ${ }^{1}$ CNR Institute of Neuroscience, Research Labs-University Milano-Bicocca, Vedano al Lambro, Italy, ${ }^{2}$ Department of Medical \\ Sciences, Section of Experimental Medicine, University of Ferrara, Ferrara, Italy
}

OPEN ACCESS

Edited by:

Francesco Caciagli,

University of Studies G.d'Annunzio

Chieti and Pescara, Italy

Reviewed by:

Fabio Grassi,

Institute for Research in Biomedicine

(IRB), Switzerland

Elisabetta Coppi,

University of Florence, Italy

${ }^{*}$ Correspondence:

Claudia Verderio

c.verderio@in.cnr.it

Specialty section:

This article was submitted to

Experimental Pharmacology and Drug

Discovery,

a section of the journal

Frontiers in Pharmacology

Received: 15 January 2021

Accepted: 09 February 2021

Published: 15 March 2021

Citation:

Lombardi M, Gabrielli M, Adinolfi E and Verderio C (2021) Role of ATP in

Extracellular Vesicle Biogenesis and Dynamics.

Front. Pharmacol. 12:654023.

doi: $10.3389 /$ fphar.2021.654023
Adenosine triphosphate (ATP) is among the molecules involved in the immune response. It acts as danger signal that promotes inflammation by activating both $\mathrm{P} 2 \mathrm{X}$ and $\mathrm{P} 2 \mathrm{Y}$ purinergic receptors expressed in immune cells, including microglia, and tumor cells. One of the most important receptors implicated in ATP-induced inflammation is P2X7 receptor (P2X7R). The stimulation of P2X7R by high concentration of ATP results in cell proliferation, inflammasome activation and shedding of extracellular vesicles (EVs). EVs are membrane structures released by all cells, which contain a selection of donor cell components, including proteins, lipids, RNA and ATP itself, and are able to transfer these molecules to target cells. ATP stimulation not only promotes EV production from microglia but also influences EV composition and signaling to the environment. In the present review, we will discuss the current knowledge on the role of ATP in the biogenesis and dynamics of EVs, which exert important functions in physiology and pathophysiology.

Keywords: ATP, extracellular vesicles, immune cells, tumor cells, P2X7 receptor, extracellular vesicle biogenesis, extracellular vesicle morphology, ATP cargo

\section{INTRODUCTION}

Adenosine triphosphate (ATP) is a ubiquitous nucleotide that not only provides energy source within cells but acts as transmitter/signaling molecule mediating interactions among various cell types in the brain (Inoue, 2002; Hansson and Ronnback, 2003) and many other organs and systems.

Under physiological conditions, the concentration of extracellular ATP (eATP) is very low (400-1,000 nM), allowing a $10^{6}$-fold gradient for ATP efflux (Trautmann, 2009). Larger increase in eATP levels occurs during metabolic stress or brain injury, and persists in the peritraumatic zone for many hours after the insult (Wang et al., 2004). Indeed, at the site of injury activated immune cells, i.e., lymphocytes (Filippini et al., 1990), macrophages (Sikora et al., 1999), microglia (Ferrari et al., 1997), and platelets (Beigi et al., 1999), release ATP and other purines, such as adenosine diphosphate (ADP) and uridine triphosphate (UTP) into the extracellular space mainly via exocytosis of secretory granules or transport through channels or transporters (Lazarowski, 2012).

\footnotetext{
Abbreviations: : ACTB, actin beta; ACTN4, actinin alpha 4; ACTN1, actinin alpha 1; ADP, adenosine diphosphate; ALDOA, aldolase A; A-SMASE, acid sphigomyelinase; ATP, adenosine triphosphate; ATP-EVs, EVs produced by ATP-stimulated microglia; Atp1a1, sodium/potassium-transporting ATPase subunit alpha-1; Atpla3, sodium/potassium-transporting ATPase subunit alpha-3; Atp5a1, ATP synthase subunit alpha; Atp5b, ATP synthase subunit beta; Atp6v1b2, V-type proton ATPase subunit B brain isoform; CFL-1, cofilin-1; CSF, cerebrospinal fluid; DAMPs, Danger Associated Molecular Patterns; Ddx25, ATP-dependent RNA helicase DDX25; eATP, extracellular ATP; ENO1, enolase-1; FLNA, filamin alpha; GAPDH, glyceraldehyde 3-phosphate dehydrogenase; GPI, glucose-6-phosphate isomerase; GSN, gelsolin; EVs, extracellular vesicles; LDHA, lactate dehydrogenase A; LDHB, lactate dehydrogenase B; MS: multiple sclerosis; PGK1, phosphoglycerate kinase 1; PM, plasma membrane; PKM: pyruvate kinase; P2XR, P2X receptors; P2X7R, P2X7 receptor; P2YR, P2Y receptors; TACE, TNF -converting enzyme; TBI, traumatic brain injury; TLN1, talin-1; TPI1, triosephosphate isomerase 1; UTP, uridine triphosphate
} 
eATP acts as a Danger Associated Molecular Patterns (DAMPs) and binds to specific surface receptors called P2 purinoceptors, promoting acute inflammation (Soni et al., 2019). There are two subsets of P2 receptors: P2Y and P2X receptors (Idzko et al., 2014). P2Y receptors (P2YR) are G-protein-coupled receptors, which mediate adenylyl cyclase, phospholipase $\mathrm{C}$ and ion channel activation (Abbracchio et al., 2006). On the contrary, $\mathrm{P} 2 \mathrm{X}$ receptors (P2XR) are $\mathrm{Ca}^{2+}$ permeable, non-selective cation channels sensitive to micromolar concentration of eATP (Trautmann, 2009). Both P2YR and P2XR are expressed on microglia, the immune cells resident in the brain, along with receptors specific for the ATP metabolite Adenosine, and are necessary for the rapid microglial response to changes in brain homeostasis (Orr et al., 2009; Illes et al., 2020).

During inflammation, microglia undergo progressive modifications, including altered expression of cell surface markers and inflammation-related genes, process retraction and acquisition of an ameboid morphology, enhanced migration and phagocytic ability (Kettenmann et al., 2011). These changes in microglial functions are partly associated with changes in purinergic receptors expression that determine different responses to ATP. Process retraction is mainly due to upregulation of adenosine receptor A2A and downregulation of P2Y12 receptors (Orr et al., 2009), whereas migration is mediated by adenosine A1 and P2X4 (Li et al., 2013) as well as P2Y12 receptors (Haynes et al., 2006; Ohsawa et al., 2007). Phagocytosis is triggered by the upregulation of P2Y6R, which is activated by the release of UTP by dying cells (Inoue, 2007). Finally, the ATP-sensitive P2X7 receptor (P2X7R) has been shown to drive important morphological alterations in microglia as well as the release of pro-inflammatory/ pathological agents via extracellular vesicles (EVs) (Ferrari et al., 2006).

The present review focuses on the role of ATP/P2X7R signaling axis in inducing EV shedding from immune and tumor cells, and on ATP involvement in the control of EV composition and dynamics of interaction with target cells.

\section{ATP STIMULATES THE RELEASE OF EVS BY IMMUNE CELLS UPON P2X7R ACTIVATION}

P2X7 receptor (P2X7R) is highly expressed on inflammatory cells (Faas et al., 2017) and requires a very high concentration $(>100 \mu \mathrm{M})$ of ATP for its activation (Trautmann, 2009). Once stimulated, influx of $\mathrm{Na}^{+}$and $\mathrm{Ca}^{2+}$ into the cell and efflux of $\mathrm{K}^{+}$ out of the cell occur, inducing cell proliferation (Nuttle and Dubyak, 1994; Bianco et al., 2006) and inflammasome activation (Yaron et al., 2015; Orioli et al., 2017). Furthermore, upon prolonged activation, P2X7R forms an aqueous pore at the cell membrane allowing the passage of hydrophilic molecules, that results in cell death (Faas et al., 2017).

Fifteen years ago, Verderio and colleagues demonstrated another fundamental function mediated by P2X7R activation in cultured microglia. P2X7R stimulation massively increases the shedding of large membrane vesicles from the plasma membrane (PM). These large extracellular vesicles (EVs), also known as microvesicles, are circular membrane structures enriched in bioactive molecules that play an important role in cell-to-cell communication (Bianco et al., 2009).

Differently from other members of the P2X family, P2X7R present a long cytoplasmic $\mathrm{C}$ terminus that contains several binding sites for Src kinases proteins, which phosphorylate and activate ROCK and p38 MAP kinases (Kanthou and Tozer, 2002; Pfeiffer et al., 2004). These signaling proteins induce the local disassembly of the cytoskeletal elements and the translocation to the PM of the enzyme acid sphigomyelinase (A-SMASE). A-SMASE hydrolyzes sphingomyelin, a phospholipid abundant in the outer leaflet of the PM, to ceramide, facilitating blebs formation and EV shedding (Figure 1A) (Bianco et al., 2009).

Notably, surface blebbing occurs in proximity of lipid rafts (Del Conde et al., 2005), where P2X7R localizes, and requires the loss of membrane asymmetry and the exposure of phosphatidylserine at the outer leaflet of PM. Vesicle shedding causes a decrease in PM capacitance (MacKenzie et al., 2001) and is markedly inhibited by removal of extracellular $\mathrm{Ca}^{2+}$ or treatment with either P2X7R antagonists (Bianco et al., 2005; Pizzirani et al., 2007) or p38 and rho kinases inhibitors (Pfeiffer et al., 2004). In accordance, membrane blebbing is increased by antagonism of the P2X7R negative regulator HSP90 (Adinolfi et al., 2003).

In addition to large EVs, P2X7R stimulation triggers release of small EVs, also called exosomes, originating in the endocytic compartment (Figure 1 Panel A) (Asai et al., 2015; Ruan et al., 2020).

The major finding related to P2X7R-dependent EV production from microglia and peripheral immune cells is linked to its involvement in the processing and release of inflammatory cytokines (MacKenzie et al., 2001; Bianco et al., 2005).

Several lines of evidence indicated that EVs are loaded with unprocessed pro-IL- $1 \beta$, mature IL- $1 \beta$ and the IL- $1 \beta$ converting enzyme caspase-1, and express $\mathrm{P} 2 \mathrm{X} 7 \mathrm{R}$ in their membranes (Bianco et al., 2005; Pizzirani et al., 2007). Caspase-1 is activated upon P2X7R stimulation on the vesicle surface, and is responsible for conversion of the biological inactive IL- $1 \beta$ precursor into the active form of the cytokine (Bianco et al., 2005) (Figures 1A,B). Other reports showed that EVs act as carriers of the protease cathepsin D (Qu et al., 2009; Sarkar et al., 2009) besides caspase-1, and other cytokines such as TNF and IL18 (Hide et al., 2000; Ferrari et al., 2006).

Specifically, Barbera-Cremades and colleagues showed that stimulation of $\mathrm{P} 2 \mathrm{X} 7 \mathrm{R}$ in macrophages leads to the release of EVs containing both TNF and the TNFconverting enzyme (TACE), that cleaves membrane-bound TNF, generating the soluble cytokine (Barbera-Cremades et al., 2017; Raffaele et al., 2020). Furthermore, a recent study demonstrated that ATP redirects TNF intracellular trafficking in activated macrophages, limiting the release of soluble TNF and preferentially packaging transmembrane TNF in EVs (Soni et al., 2019). Importantly, TNF-carrying 

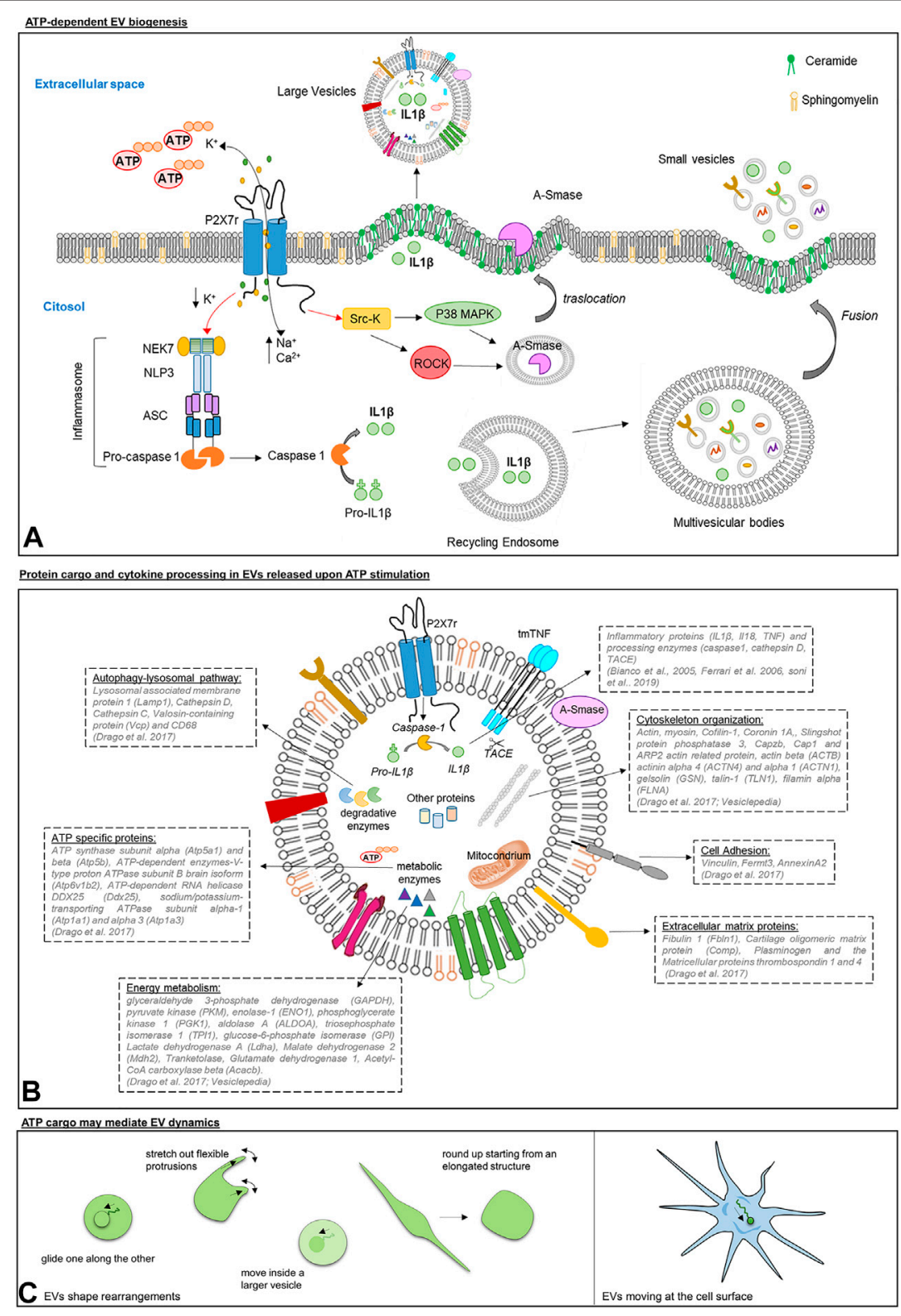

FIGURE 1 | Panel (A): Scheme of ATP/P2X7R signaling axis inducing EV shedding from immune cells. Upon ATP stimulation, P2X7R activates p38 MAPK and ROCK through Src kinases. In turn, $p 38$ and ROCK trigger the local disassembly of the cytoskeletal elements and the mobilization of A-SMASE from the luminal lysosomal compartment to the outer leaflet of the PM, where the enzyme hydrolyzes sphingomyelin to ceramide favoring blebs formation and the shedding of large vesicles carrying IL-1 $\beta$ (Bianco et al., 2005).P2X7R also regulates the release of small EVs. ATP-induced P2X7R stimulation drives the assembly and the activation of the inflammasome composed by regulatory proteins, NEK7, ASC and NLRP3, which are essential for caspase-1 activation. Caspase 1 is a protease implicated in IL-1 $\beta$ processing and in regulating the membrane trafficking pathways that control multivesicular bodies fusion with the PM and the release of IL-1 $\beta$ storing small EVs (Qu et al., 2009). Panel (B): Schematic representation of protein cargo and cytokine processing in large EVs released upon ATP stimulation. By activating P2X7R, ATP induces in large EVs the processing of inflammatory cytokines (Hide et al., 2000; Bianco et al., 2005; Ferrari et al., 2006) and sorting of proteins implicated in autophagy-lysosomal pathway, phagocytosis and endocytosis, energy metabolism and cell adhesion/extracellular matrix organization (Drago et al., 2017). Panel (C): Graphic representation of morphological changes of EVs isolated from human mast cell lines, human blood serum, mouse lung, and Saccharomyces cerevisiae as imaged by Cvjetkovic and colleagues (Cvjetkovic et al., 2017) (left), and of a single EV in motion at the cell surface of microglia (Prada et al., 2016) (right).

EVs are biologically more potent than soluble TNF, and mediate significant lung inflammation in mice (Soni et al., 2019).

Through EV production, inflammatory proteins can be released at significant distance from donor cell, in possible proximity to target cells, thus preventing the dispersal and degradation of mediators in the extracellular environment.

In recent years, several studies investigated the physiological and pathological functions of EVs within the brain. These functions include control of neuronal development (Marzesco 
et al., 2005), synaptic activity (Antonucci et al., 2012; Gabrielli et al., 2015), axon-glial transfer of information (Prada et al., 2018), nerve regeneration (Lai and Breakefield, 2012) and myelin formation (Pusic et al., 2016; Van Niel et al., 2018; Lombardi et al., 2019); as well as disease-associated events, such as tumor progression, spreading of inflammation (Verderio et al., 2012) or dissemination of pathogenic proteins (Joshi et al., 2014; Asai et al., 2015; Eitan et al., 2016; Sardar Sinha et al., 2018; Crotti et al., 2019; Ruan et al., 2020). However, the contribution of ATPinduced EV shedding vs. constitutive EV release in brain disease pathogenesis is just emerging.

ATP-induced EVs may play a relevant pathogenic role in traumatic brain injury (TBI). After TBI, high concentrations of eATP activate P2X7R in microglia and increase EV production, while treatment of TBI-affecting rats with the P2X7R antagonist A804598 or the immune modulator FTY720, that inhibits A-SMase-dependent EV biogenesis (Verderio et al., 2012), significantly decreases the number of microglial EVs in the injured/adjacent regions and in cerebrospinal fluid (CSF), and improves disease outcome (Liu et al., 2017).

Another study implicated microglial EVs released upon ATP stimulation of P2X7R in the spreading of tau protein and disease progression in a tauopathy mouse model. Specifically, pharmacologic blockade of P2X7R with GSK1482160, an orally applicable and CNS-penetrant inhibitor, suppressed both secretion of small EVs (exosomes) and disease outcome in the early disease stages (Ruan et al., 2020).

Conversely, the role of ATP and P2X7R-mediated EV release in multiple sclerosis (MS), the prototypical neuroinflammatory disease, remains controversial. Treatment of EAE mice, a MS mouse model, with the specific P2X7R antagonists oxATP and BBG reduced disease severity (Matute et al., 2007), but P2X7R knockout mice displayed a more severe pathology (Chen and Brosnan, 2006). Furthermore, EAE ameliorated in A-SMASE knock-out mice, genetically impaired in ATP-evoked EV production (Verderio et al., 2012), but injection of the A-SMASE inhibitor imipramine did not significantly reduce the level of myeloid EVs in the CSF. The latter finding rules out a major role of ATP in sustaining EV production in a context of chronic neuroinflammation, where cytokines may mainly control EV release from myeloid cells (Colombo et al., 2018).

\section{P2X7R-DEPENDENT EV PRODUCTION FROM TUMOR CELLS}

The tumor microenvironment is rich in eATP, and the role of this nucleotide and its receptors, particularly P2X7R, in cancer has been the focus of numerous papers in recent years (Di Virgilio et al., 2018; Adinolfi et al., 2019; Lara et al., 2020). P2X7R is upregulated in solid cancer and onco-hematological conditions, and several preclinical studies have demonstrated that its blockade has good potential as an anticancer treatment (Adinolfi et al., 2012; De Marchi et al., 2019; Pegoraro et al., 2020). Recently, the association of P2X7R with EV release has been supported by a work showing an increase in cancer patient's serum concentration of soluble P2X7R, possibly expressed on the surface of EVs (Giuliani et al., 2019). As mentioned above, the activation of P2X7R is also associated with the release of EVs from the monocyte/macrophage cell lineage (Baroni et al., 2007; Pizzirani et al., 2007; Gulinelli et al., 2012). These vesicles carry several molecules, including cytokines and tissue factor, associated with cancer pathogenesis and progression but also with tumor immune eradication and immunosuppression (Graner, 2018). EVs released from both cancer and immune cells have shown to facilitate angiogenesis, cause extracellular matrix remodeling, prepare the pre-metastatic niche, and consequently cause organ tropism of disseminating tumor cells (Kuriyama et al., 2020; Schubert and Boutros, 2021). However, only few manuscripts have reported P2X7-dependent EV release from cancer cells (Gutierrez-Martin et al., 2011; Kholia et al., 2015; Park et al., 2019) and therefore evidence relating to P2X7R activity, EV content, and cancer function is far to be complete and will deserve further attention.

\section{ATP STIMULATION INFLUENCES EV COMPOSITION}

So far, only one study showed that ATP strongly influences the composition of EVs (Drago et al., 2017). Label free proteomics revealed that ATP stimulation induces sorting into microglial EVs of proteins implicated in autophagy-lysosomal pathway, phagocytosis and endocytosis, energy metabolism and cell adhesion/extracellular matrix organization (Drago et al., 2017).

The overexpression of degradative enzymes in EVs produced by ATP-stimulated microglia (ATP-EVs), compared to those released constitutively, may reflect the enhanced capacity of microglia to phagocyte apoptotic cells or synapses in response to ATP. By contrast, the abundance of metabolic enzymes necessary for glycolysis, lactate production, the oxidative branch of the pentose phosphate pathway, glutamine metabolism and fatty acid synthesis may reflect an increase in cellular metabolism to sustain ATP-dependent microglial functions, such as process scanning and phagocytic activity (Grabert et al., 2016).

Due to the higher content of proteins involved in extracellular matrix organization and cell adhesion, ATP-EVs adhere more and have stronger capacity to activate cultured astrocytes compared to constitutive EVs.

Collectively, these data indicate that ATP stimulation not only promotes EV production from microglia but also enhances their signaling to the environment.

\section{ATP IS AMONG THE CARGO OF EVS}

ATP is a component of EVs (Graner, 2018). A pioneer study by Ronquist and colleagues showed that small EVs generated in the endocytic compartment of prostate epithelial cells, also called prostasomes, can produce ATP by glycolysis. Prosteasomes contain glycolytic enzymes and their capacity to produce ATP from fructose or glucose has been proven by the luciferin/ luciferase assay (Ronquist et al., 2013). Interestingly, glycolytic 
enzymes, generating ATP from glucose, have been systematically reported in EVs of different cell origin, including EVs produced by mesenchymal stem cells, which have the capacity to restore ATP levels when delivered to an ischemic tissue (Arslan et al., 2013). Among glycolytic enzymes, 3-phosphate dehydrogenase (GAPDH), pyruvate kinase (PKM), enolase-1 (ENO1), phosphoglycerate kinase 1 (PGK1), aldolase A (ALDOA), triosephosphate isomerase 1 (TPI1) and glucose-6-phosphate isomerase (GPI) are listed among the 100 proteins more often identified in EVs according to the database Vesiclepedia (http:// microvesicles.org/), suggesting that ATP production by glycolysis may be a common feature of EVs (Figure 1B).

Furthermore, it has been recently shown that mitochondria, the main source of cellular ATP, can be also packaged into EVs (Figure 1B) (Hough et al., 2018; Zhang et al., 2020), further indicating that ATP can be generated in metabolically active EVs.

\section{ATP CARGO MAY INFLUENCE THE DYNAMICS OF EV INTERACTION WITH RECIPIENT CELLS}

Inside EVs ATP may represent a crucial source of energy, able to fuel active processes, such as the activity of ATP-dependent enzymes, e.g., V-type proton ATPase subunit B (Atp6v1b2), RNA helicase DDX25 (Ddx25), Sodium/potassiumtransporting ATPase subunit alpha-1 and 3 (Atp1a1, Atp1a3), which are part of the proteome of microglial EVs (Figure 1B) (Drago et al., 2017). More importantly, vesicular ATP may support cytoskeleton rearrangements. Consistently, a large body of evidence has located actin inside EVs, a key component of the cellular cytoskeleton mediating cell migration and shape changes, and allowing cells to form adhesion with each other and with the extracellular matrix (Figures 1A,C) (Svitkina, 2018). Cryo-electron micrographs imaged actin-like filaments in a subpopulation of EVs isolated from different biological samples [fresh plasma, (Yuana et al., 2013); human ejaculate, (Hoog and Lotvall, 2015); human ejaculate and human mast cell cultures, (Cvjetkovic et al., 2017); HeLa cells, (Yang et al., 2020)] and its presence was confirmed by mass-spectrometry, western blot analysis or mRNA Microarray in EVs from most cell types (human dendritic cells, (Kowal et al., 2016); mouse microglia, (Drago et al., 2017); mesenchymal stem cells, (Adamo et al., 2019); HeLa cells, (Yang et al., 2020); osteoclasts, (Holliday et al., 2019); human blood, (Eguchi et al., 2020), and more), together with actin-binding proteins and regulators of actin cytoskeleton. Not surprisingly, actin beta (ACTB) and several actin network proteins (such as actinin alpha 4 (ACTN4) and alpha 1 (ACTN1), gelsolin (GSN), cofilin-1 (CFL1), talin-1 (TLN1), filamin alpha (FLNA)) are among the top 100 proteins more frequently detected in EVs on Vesiclepedia (Figure 1B Panel B). These data open up the fascinating possibility that EVs, exploiting actin complexes present in their lumen and ATP as energy source, may have an intrinsic capacity to change their shape to interact with target cells.
In support to this hypothesis, findings from Jan Lötvall's and Johanna L Höög's laboratories have shown that, among EVs either isolated from cell cultures, biological fluids or tissue, a part (albeit small) displays morphological changes detectable by timelapse fluorescence imaging Cvjetkovic et al., 2017. EVs could round up starting from an elongated structure, glide one along the other, move inside a larger vesicle and, importantly, stretch out flexible protrusions (Figure 1C). Being exhibited also by EVs from Saccharomyces cerevisiae, these phenomena seem to be evolutionary conserved. Furthermore, time-lapse imaging revealed that single EVs, produced by microglia and gently placed in contact with other microglial cells by optical manipulation, can move after adhesion along the cell surface toward sites of internalization (Figure 1C) (Prada et al., 2016). These findings point at the possibility for EVs to undergo an ATP-dependent actin-mediated form of extracellular motion. Further experiments will be necessary to verify this captivating hypothesis. Intriguingly, intrinsic active motility would allow EVs to travel in the extracellular space at the cell surface, independently from fluid fluxes or cell-driven mechanisms (e.g., filopodia surfing/grabbing/pushing, (Heusermann et al., 2016), and we can speculate that it might even ease cell entry at specific sites of the PM. These perspectives are absolutely worth to be better explored in the future.

\section{CONCLUSION}

The interest for EVs released upon ATP stimulation has increased exponentially, given that they have become vehicle of inflammatory signals (Verderio et al., 2012), tumorigenic factors (Graner, 2018) or misfolded proteins in neurodegenerative diseases (Ruan et al., 2020), and their number is significantly augmented in the body fluids of patients affected by many neurological diseases (Verderio et al., 2012; Colombo et al., 2018). Since the analysis of EV cargo may provide indications on the activation state of donor cells and the pathological state of the brain, EVs are currently under intense investigation for a possible employment in clinical practice as prognostic biomarkers. In addition, further knowledge of $\mathrm{EV}$ dynamics and interaction with target cells may reveal new molecular targets to limit cancer metastasis and propagation of neurodegenerative lesions throughout the brain.

\section{AUTHOR CONTRIBUTIONS}

All authors contributed to the writing and final approval of the manuscript.

\section{FUNDING}

This study was funded by Ministero della salute, Ricerca Finalizzata-2016-02361492 to CV. 


\section{REFERENCES}

Abbracchio, M. P., Burnstock, G., Boeynaems, J. M., Barnard, E. A., Boyer, J. L., Kennedy, C., et al. (2006). International union of pharmacology LVIII: update on the P2Y G protein-coupled nucleotide receptors: from molecular mechanisms and pathophysiology to therapy. Pharmacol. Rev. 58, 281-341. doi:10.1124/pr.58.3.3

Adamo, A., Brandi, J., Caligola, S., Delfino, P., Bazzoni, R., Carusone, R., et al. (2019). Extracellular vesicles mediate mesenchymal stromal cell-dependent regulation of $\mathrm{B}$ cell $\mathrm{PI} 3 \mathrm{~K}-\mathrm{AKT}$ signaling pathway and actin cytoskeleton. Front. Immunol. 10, 446. doi:10.3389/fimmu.2019.00446

Adinolfi, E., De Marchi, E., Orioli, E., Pegoraro, A., and Di Virgilio, F. (2019). Role of the P2X7 receptor in tumor-associated inflammation. Curr. Opin. Pharmacol. 47, 59-64. doi:10.1016/j.coph.2019.02.012

Adinolfi, E., Kim, M., Young, M. T., Di Virgilio, F., and Surprenant, A. (2003). Tyrosine phosphorylation of HSP90 within the P2X7 receptor complex negatively regulates P2X7 receptors. J. Biol. Chem. 278, 37344-37351. doi:10.1074/jbc.M301508200

Adinolfi, E., Raffaghello, L., Giuliani, A. L., Cavazzini, L., Capece, M., Chiozzi, P., et al. (2012). Expression of P2X7 receptor increases in vivo tumor growth. Cancer Res. 72, 2957-2969. doi:10.1158/0008-5472.CAN-11-1947

Antonucci, F., Turola, E., Riganti, L., Caleo, M., Gabrielli, M., Perrotta, C., et al. (2012). Microvesicles released from microglia stimulate synaptic activity via enhanced sphingolipid metabolism. EMBO J. 31, 1231-1240. doi:10.1038/ emboj.2011.489

Arslan, F., Lai, R. C., Smeets, M. B., Akeroyd, L., Choo, A., Aguor, E. N., et al. (2013). Mesenchymal stem cell-derived exosomes increase ATP levels, decrease oxidative stress and activate PI3K/Akt pathway to enhance myocardial viability and prevent adverse remodeling after myocardial ischemia/reperfusion injury. Stem Cell Res. 10, 301-312. doi:10.1016/j.scr.2013.01.002

Asai, H., Ikezu, S., Tsunoda, S., Medalla, M., Luebke, J., Haydar, T., et al. (2015). Depletion of microglia and inhibition of exosome synthesis halt tau propagation. Nat. Neurosci. 18, 1584-1593. doi:10.1038/nn.4132

Barbera-Cremades, M., Gomez, A. I., Baroja-Mazo, A., Martinez-Alarcon, L., Martinez, C. M., De Torre-Minguela, C., et al. (2017). P2X7 receptor induces tumor necrosis factor-alpha converting enzyme activation and release to boost TNF-alpha production. Front. Immunol. 8, 862. doi:10. 3389/fimmu.2017.00862

Baroni, M., Pizzirani, C., Pinotti, M., Ferrari, D., Adinolfi, E., Calzavarini, S., et al. (2007). Stimulation of P2 (P2X7) receptors in human dendritic cells induces the release of tissue factor-bearing microparticles. FASEB J. 21, 1926-1933. doi:10. 1096/fj.06-7238com

Beigi, R., Kobatake, E., Aizawa, M., and Dubyak, G. R. (1999). Detection of local ATP release from activated platelets using cell surface-attached firefly luciferase. Am. J. Physiol. 276, C267-C278. doi:10.1152/ajpcell.1999.276.1.C267

Bianco, F., Ceruti, S., Colombo, A., Fumagalli, M., Ferrari, D., Pizzirani, C., et al. (2006). A role for P2X7 in microglial proliferation. J. Neurochem. 99, 745-758. doi:10.1111/j.1471-4159.2006.04101.x

Bianco, F., Perrotta, C., Novellino, L., Francolini, M., Riganti, L., Menna, E., et al. (2009). Acid sphingomyelinase activity triggers microparticle release from glial cells. EMBO J. 28, 1043-1054. doi:10.1038/emboj.2009.45

Bianco, F., Pravettoni, E., Colombo, A., Schenk, U., Moller, T., Matteoli, M., et al. (2005). Astrocyte-derived ATP induces vesicle shedding and IL-1 beta release from microglia. J. Immunol. 174, 7268-7277. doi:10.4049/jimmunol.174.11. 7268

Chen, L., and Brosnan, C. F. (2006). Exacerbation of experimental autoimmune encephalomyelitis in $\mathrm{P} 2 \mathrm{X} \mathrm{R}^{-/-}$mice: evidence for loss of apoptotic activity in lymphocytes. J. Immunol. 176, 3115-3126. doi:10.4049/jimmunol.176.5. 3115

Colombo, F., Bastoni, M., Nigro, A., Podini, P., Finardi, A., Casella, G., et al. (2018). Cytokines stimulate the release of microvesicles from myeloid cells independently from the $\mathrm{P} 2 \mathrm{X} 7$ receptor/acid sphingomyelinase pathway. Front. Immunol. 9, 204. doi:10.3389/fimmu.2018.00204

Crotti, A., Sait, H. R., Mcavoy, K. M., Estrada, K., Ergun, A., Szak, S., et al. (2019). BIN1 favors the spreading of Tau via extracellular vesicles. Sci. Rep. 9, 9477. doi:10.1038/s41598-019-45676-0
Cvjetkovic, A., Crescitelli, R., Lässer, C., Zabeo, D., Widlund, P., Nyström, T., et al. (2017). Extracellular vesicles in motion. Matters 784, doi:10.19185/matters. 201704000003

De Marchi, E., Orioli, E., Pegoraro, A., Sangaletti, S., Portararo, P., Curti, A., et al. (2019). The P2X7 receptor modulates immune cells infiltration, ectonucleotidases expression and extracellular ATP levels in the tumor microenvironment. Oncogene 38, 3636-3650. doi:10.1038/s41388-019-0684-y

Del Conde, I., Shrimpton, C. N., Thiagarajan, P., and Lopez, J. A. (2005). Tissuefactor-bearing microvesicles arise from lipid rafts and fuse with activated platelets to initiate coagulation. Blood 106, 1604-1611. doi:10.1182/blood2004-03-1095

Di Virgilio, F., Sarti, A. C., Falzoni, S., De Marchi, E., and Adinolfi, E. (2018). Extracellular ATP and P2 purinergic signalling in the tumour microenvironment. Nat. Rev. Cancer 18, 601-618. doi:10.1038/s41568-0180037-0

Drago, F., Lombardi, M., Prada, I., Gabrielli, M., Joshi, P., Cojoc, D., et al. (2017). ATP modifies the proteome of extracellular vesicles released by microglia and influences their action on astrocytes. Front. Pharmacol. 8, 910. doi:10.3389/ fphar.2017.00910

Eguchi, A., Fukuda, S., Kuratsune, H., Nojima, J., Nakatomi, Y., Watanabe, Y., et al. (2020). Identification of actin network proteins, talin-1 and filamin-A, in circulating extracellular vesicles as blood biomarkers for human myalgic encephalomyelitis/chronic fatigue syndrome. Brain Behav. Immun. 84, 106-114. doi:10.1016/j.bbi.2019.11.015

Eitan, E., Suire, C., Zhang, S., and Mattson, M. P. (2016). Impact of lysosome status on extracellular vesicle content and release. Ageing Res. Rev. 32, 65-74. doi:10. 1016/j.arr.2016.05.001

Faas, M. M., Saez, T., and De Vos, P. (2017). Extracellular ATP and adenosine: the Yin and Yang in immune responses? Mol. Aspects Med. 55, 9-19. doi:10.1016/j. mam.2017.01.002

Ferrari, D., Chiozzi, P., Falzoni, S., Dal Susino, M., Collo, G., Buell, G., et al. (1997). ATP-mediated cytotoxicity in microglial cells. Neuropharmacology 36, 1295-1301. doi:10.1016/s0028-3908(97)00137-8

Ferrari, D., Pizzirani, C., Adinolfi, E., Lemoli, R. M., Curti, A., Idzko, M., et al. (2006). The P2X7 receptor: a key player in IL-1 processing and release. J. Immunol. 176, 3877-3883. doi:10.4049/jimmunol.176.7.3877

Filippini, A., Taffs, R. E., and Sitkovsky, M. V. (1990). Extracellular ATP in T-lymphocyte activation: possible role in effector functions. Proc. Natl. Acad. Sci. U.S.A. 87, 8267-8271. doi:10.1073/pnas.87.21.8267

Gabrielli, M., Battista, N., Riganti, L., Prada, I., Antonucci, F., Cantone, L., et al. (2015). Active endocannabinoids are secreted on extracellular membrane vesicles. EMBO Rep. 16, 213-220. doi:10.15252/embr.201439668

Giuliani, A. L., Berchan, M., Sanz, J. M., Passaro, A., Pizzicotti, S., Vultaggio-Poma, V., et al. (2019). The P2X7 receptor is shed into circulation: correlation with C-reactive protein levels. Front. Immunol. 10, 793. doi:10.3389/fimmu.2019. 00793

Grabert, K., Michoel, T., Karavolos, M. H., Clohisey, S., Baillie, J. K., Stevens, M. P., et al. (2016). Microglial brain region-dependent diversity and selective regional sensitivities to aging. Nat. Neurosci. 19, 504-516. doi:10.1038/nn.4222

Graner, M. W. (2018). Extracellular vesicles in cancer immune responses: roles of purinergic receptors. Semin. Immunopathol. 40, 465-475. doi:10.1007/s00281018-0706-9

Gulinelli, S., Salaro, E., Vuerich, M., Bozzato, D., Pizzirani, C., Bolognesi, G., et al. (2012). IL-18 associates to microvesicles shed from human macrophages by a LPS/TLR-4 independent mechanism in response to P2X receptor stimulation. Eur. J. Immunol. 42, 3334-3345. doi:10.1002/eji.201142268

Gutierrez-Martin, Y., Bustillo, D., Gomez-Villafuertes, R., Sanchez-Nogueiro, J., Torregrosa-Hetland, C., Binz, T., et al. (2011). P2X7 receptors trigger ATP exocytosis and modify secretory vesicle dynamics in neuroblastoma cells. J. Biol. Chem. 286, 11370-11381. doi:10.1074/jbc.M110.139410

Hansson, E., and Ronnback, L. (2003). Glial neuronal signaling in the central nervous system. FASEB J. 17, 341-348. doi:10.1096/fj.02-0429rev

Haynes, S. E., Hollopeter, G., Yang, G., Kurpius, D., Dailey, M. E., Gan, W. B., et al. (2006). The P2Y12 receptor regulates microglial activation by extracellular nucleotides. Nat. Neurosci. 9, 1512-1519. doi:10.1038/nn1805

Heusermann, W., Hean, J., Trojer, D., Steib, E., Von Bueren, S., Graff-Meyer, A., et al. (2016). Exosomes surf on filopodia to enter cells at endocytic hot spots, 
traffic within endosomes, and are targeted to the ER. J. Cell Biol. 213, 173-184. doi:10.1083/jcb.201506084

Hide, I., Tanaka, M., Inoue, A., Nakajima, K., Kohsaka, S., Inoue, K., et al. (2000). Extracellular ATP triggers tumor necrosis factor-alpha release from rat microglia. J. Neurochem. 75, 965-972. doi:10.1046/j.1471-4159.2000.0750965.x

Holliday, L. S., Faria, L. P., and Rody, W. J., Jr (2019). Actin and actin-associated proteins in extracellular vesicles shed by osteoclasts. Int. J. Mol. Sci. 21, 158. doi:10.3390/ijms21010158

Hoog, J. L., and Lotvall, J. (2015). Diversity of extracellular vesicles in human ejaculates revealed by cryo-electron microscopy. J. Extracell. Vesicles 4, 28680. doi: $10.3402 /$ jev.v4.28680

Hough, K. P., Trevor, J. L., Strenkowski, J. G., Wang, Y., Chacko, B. K., Tousif, S., et al. (2018). Exosomal transfer of mitochondria from airway myeloid-derived regulatory cells to T cells. Redox Biol. 18, 54-64. doi:10.1016/j.redox.2018. 06.009

Idzko, M., Ferrari, D., and Eltzschig, H. K. (2014). Nucleotide signalling during inflammation. Nature 509, 310-317. doi:10.1038/nature13085

Illes, P., Rubini, P., Ulrich, H., Zhao, Y., and Tang, Y. (2020). Regulation of microglial functions by purinergic mechanisms in the healthy and diseased CNS. Cells 9, 1108. doi:10.3390/cells9051108

Inoue, K. (2002). Microglial activation by purines and pyrimidines. Glia 40, 156-163. doi:10.1002/glia.10150

Inoue, K. (2007). UDP facilitates microglial phagocytosis through P2Y6 receptors. Cell Adh. Migr. 1, 131-132. doi:10.4161/cam.1.3.4937

Joshi, P., Turola, E., Ruiz, A., Bergami, A., Libera, D. D., Benussi, L., et al. (2014). Microglia convert aggregated amyloid-beta into neurotoxic forms through the shedding of microvesicles. Cell Death Differ. 21, 582-593. doi:10.1038/cdd. 2013.180

Kanthou, C., and Tozer, G. M. (2002). The tumor vascular targeting agent combretastatin A-4-phosphate induces reorganization of the actin cytoskeleton and early membrane blebbing in human endothelial cells. Blood 99, 2060-2069. doi:10.1182/blood.v99.6.2060

Kettenmann, H., Hanisch, U. K., Noda, M., and Verkhratsky, A. (2011). Physiology of microglia. Physiol. Rev. 91, 461-553. doi:10.1152/physrev.00011.2010

Kholia, S., Jorfi, S., Thompson, P. R., Causey, C. P., Nicholas, A. P., Inal, J. M., et al. (2015). A novel role for peptidylarginine deiminases in microvesicle release reveals therapeutic potential of PAD inhibition in sensitizing prostate cancer cells to chemotherapy. J. Extracell. Vesicles 4, 26192. doi:10.3402/jev.v4.26192

Kowal, J., Arras, G., Colombo, M., Jouve, M., Morath, J. P., Primdal-Bengtson, B., et al. (2016). Proteomic comparison defines novel markers to characterize heterogeneous populations of extracellular vesicle subtypes. Proc. Natl. Acad. Sci. U.S.A. 113, E968-E977. doi:10.1073/pnas.1521230113

Kuriyama, N., Yoshioka, Y., Kikuchi, S., Azuma, N., and Ochiya, T. (2020). Extracellular vesicles are key regulators of tumor neovasculature. Front. Cell Dev. Biol. 8, 611039. doi:10.3389/fcell.2020.611039

Lai, C. P., and Breakefield, X. O. (2012). Role of exosomes/microvesicles in the nervous system and use in emerging therapies. Front. Physiol. 3, 228. doi:10. 3389/fphys.2012.00228

Lara, R., Adinolfi, E., Harwood, C. A., Philpott, M., Barden, J. A., Di Virgilio, F., et al. (2020). P2X7 in cancer: from molecular mechanisms to therapeutics. Front. Pharmacol. 11, 793. doi:10.3389/fphar.2020.00793

Lazarowski, E. R. (2012). Vesicular and conductive mechanisms of nucleotide release. Purinergic Signal. 8, 359-373. doi:10.1007/s11302-012-9304-9

Li, Z., Li, W., Li, Q., and Thang, M. (2013). Extracellular nucleotides and adenosine regulate microglial motility and their role in cerebral ischemia Acta Pharm. Sin. B 3, 205. doi:10.1016/j.apsb.2013.06.003

Liu, X., Zhao, Z., Ji, R., Zhu, J., Sui, Q. Q., Knight, G. E., et al. (2017). Inhibition of P2X7 receptors improves outcomes after traumatic brain injury in rats. Purinergic Signal 13, 529-544. doi:10.1007/s11302-017-9579-y

Lombardi, M., Parolisi, R., Scaroni, F., Bonfanti, E., Gualerzi, A., Gabrielli, M., et al. (2019). Detrimental and protective action of microglial extracellular vesicles on myelin lesions: astrocyte involvement in remyelination failure. Acta Neuropathol. 138, 987-1012.doi:10.1007/s00401-019-02049-1

Mackenzie, A., Wilson, H. L., Kiss-Toth, E., Dower, S. K., North, R. A., and Surprenant, A. (2001). Rapid secretion of interleukin-1beta by microvesicle shedding. Immunity 15, 825-835. doi:10.1016/s1074-7613(01)00229-1

Marzesco, A. M., Janich, P., Wilsch-Brauninger, M., Dubreuil, V., Langenfeld, K., Corbeil, D., et al. (2005). Release of extracellular membrane particles carrying the stem cell marker prominin-1 (CD133) from neural progenitors and other epithelial cells. J. Cell Sci. 118, 2849-2858. doi:10.1242/jcs.02439

Matute, C., Torre, I., Perez-Cerda, F., Perez-Samartin, A., Alberdi, E., Etxebarria, E., et al. (2007). P2X(7) receptor blockade prevents ATP excitotoxicity in oligodendrocytes and ameliorates experimental autoimmune encephalomyelitis. J. Neurosci. 27, 9525-9533. doi:10.1523/JNEUROSCI. 0579-07.2007

Nuttle, L. C., and Dubyak, G. R. (1994). Differential activation of cation channels and non-selective pores by macrophage $\mathrm{P} 2 \mathrm{z}$ purinergic receptors expressed in Xenopus oocytes. J. Biol. Chem. 269, 13988-13996. doi:10.1016/s0021-9258(17) 36744-3

Ohsawa, K., Irino, Y., Nakamura, Y., Akazawa, C., Inoue, K., and Kohsaka, S. (2007). Involvement of P2X 4 and P2Y12 receptors in ATP-induced microglial chemotaxis. Glia 55, 604-616. doi:10.1002/glia.20489

Orioli, E., De Marchi, E., Giuliani, A. L., and Adinolfi, E. (2017). P2X7 receptor orchestrates multiple signalling pathways triggering inflammation, autophagy and metabolic/trophic responses. Curr. Med. Chem. 24, 2261-2275. doi:10. 2174/0929867324666170303161659

Orr, A. G., Orr, A. L., Li, X. J., Gross, R. E., and Traynelis, S. F. (2009). Adenosine $\mathrm{A}(2 \mathrm{~A})$ receptor mediates microglial process retraction. Nat. Neurosci. 12, 872-878. doi:10.1038/nn.2341

Park, M., Kim, J., Phuong, N. T. T., Park, J. G., Park, J. H., Kim, Y. C., et al. (2019). Involvement of the $\mathrm{P} 2 \mathrm{X} 7$ receptor in the migration and metastasis of tamoxifen-resistant breast cancer: effects on small extracellular vesicles production. Sci. Rep. 9, 11587. doi:10.1038/s41598-019-47734-z

Pegoraro, A., Orioli, E., De Marchi, E., Salvestrini, V., Milani, A., Di Virgilio, F., et al. (2020). Differential sensitivity of acute myeloid leukemia cells to daunorubicin depends on P2X7A versus P2X7B receptor expression. Cell Death Dis. 11, 876. doi:10.1038/s41419-020-03058-9

Pfeiffer, Z. A., Aga, M., Prabhu, U., Watters, J. J., Hall, D. J., and Bertics, P. J. (2004). The nucleotide receptor $\mathrm{P} 2 \mathrm{X} 7$ mediates actin reorganization and membrane blebbing in RAW 264.7 macrophages via p38 MAP kinase and Rho. J. Leukoc. Biol. 75, 1173-1182. doi:10.1189/jlb.1203648

Pizzirani, C., Ferrari, D., Chiozzi, P., Adinolfi, E., Sandona, D., Savaglio, E., et al. (2007). Stimulation of P2 receptors causes release of IL-1beta-loaded microvesicles from human dendritic cells. Blood 109, 3856-3864. doi:10. 1182/blood-2005-06-031377

Prada, I., Amin, L., Furlan, R., Legname, G., Verderio, C., and Cojoc, D. (2016). A new approach to follow a single extracellular vesicle-cell interaction using optical tweezers. Biotechniques 60, 35-41. doi:10.2144/000114371

Prada, I., Gabrielli, M., Turola, E., Iorio, A., D’arrigo, G., Parolisi, R., et al. (2018). Glia-to-neuron transfer of miRNAs via extracellular vesicles: a new mechanism underlying inflammation-induced synaptic alterations. Acta Neuropathol. 135, 529-550. doi:10.1007/s00401-017-1803-x

Pusic, K. M., Pusic, A. D., and Kraig, R. P. (2016). Environmental enrichment stimulates immune cell secretion of exosomes that promote CNS myelination and may regulate inflammation. Cell Mol. Neurobiol. 36, 313-325. doi:10.1007/ s10571-015-0269-4

Qu, Y., Ramachandra, L., Mohr, S., Franchi, L., Harding, C. V., Nunez, G., et al. (2009). P2X7 receptor-stimulated secretion of MHC class IIcontaining exosomes requires the ASC/NLRP3 inflammasome but is independent of caspase-1. J. Immunol. 182, 5052-5062. doi:10.4049/ jimmunol.0802968

Raffaele, S., Lombardi, M., Verderio, C., and Fumagalli, M. (2020). TNF production and release from microglia via extracellular vesicles: impact on brain functions. Cells 9, 2145. doi:10.3390/cells9102145

Ronquist, K. G., Ek, B., Stavreus-Evers, A., Larsson, A., and Ronquist, G. (2013). Human prostasomes express glycolytic enzymes with capacity for ATP production. Am. J. Physiol. Endocrinol. Metab. 304, E576-E582. doi:10.1152/ ajpendo.00511.2012

Ruan, Z., Delpech, J. C., Venkatesan Kalavai, S., Van Enoo, A. A., Hu, J., Ikezu, S., et al. (2020). P2RX7 inhibitor suppresses exosome secretion and disease phenotype in P301S tau transgenic mice. Mol. Neurodegener. 15, 47. doi:10. 1186/s13024-020-00396-2

Sardar Sinha, M., Ansell-Schultz, A., Civitelli, L., Hildesjo, C., Larsson, M., Lannfelt, L., et al. (2018). Alzheimer's disease pathology propagation by exosomes containing toxic amyloid-beta oligomers. Acta Neuropathol. 136, 41-56. doi:10.1007/s00401-018-1868-1 
Sarkar, A., Mitra, S., Mehta, S., Raices, R., and Wewers, M. D. (2009). Monocyte derived microvesicles deliver a cell death message via encapsulated caspase-1. PLoS One 4, e7140. doi:10.1371/journal.pone.0007140

Schubert, A., and Boutros, M. (2021). Extracellular vesicles and oncogenic signaling. Mol. Oncol. 15, 3-26. doi:10.1002/1878-0261.12855

Sikora, A., Liu, J., Brosnan, C., Buell, G., Chessel, I., and Bloom, B. R. (1999). Cutting edge: purinergic signaling regulates radical-mediated bacterial killing mechanisms in macrophages through a P2X7-independent mechanism. J. Immunol. 163, 558-561.

Soni, S., O'dea, K. P., Tan, Y. Y., Cho, K., Abe, E., Romano, R., et al. (2019). ATP redirects cytokine trafficking and promotes novel membrane TNF signaling via microvesicles. FASEB J. 33, 6442-6455. doi:10.1096/fj.201802386R

Svitkina, T. (2018). The actin cytoskeleton and actin-based motility. Cold Spring Harb. Perspect. Biol. 10, a018267. doi:10.1101/cshperspect.a018267

Trautmann, A. (2009). Extracellular ATP in the immune system: more than just a “danger signal”. Sci. Signal. 2, pe6. doi:10.1126/scisignal.256pe6

Van Niel, G., D'angelo, G., and Raposo, G. (2018). Shedding light on the cell biology of extracellular vesicles. Nat. Rev. Mol. Cell Biol. 19, 213-228. doi:10. 1038/nrm.2017.125

Verderio, C., Muzio, L., Turola, E., Bergami, A., Novellino, L., Ruffini, F., et al. (2012). Myeloid microvesicles are a marker and therapeutic target for neuroinflammation. Ann. Neurol. 72, 610-624. doi:10.1002/ana.23627

Wang, X., Arcuino, G., Takano, T., Lin, J., Peng, W. G., Wan, P., et al. (2004). P2X7 receptor inhibition improves recovery after spinal cord injury. Nat. Med. 10, 821-827. doi:10.1038/nm1082

Yang, J. E., Rossignol, E. D., Chang, D., Zaia, J., Forrester, I., Raja, K., et al. (2020). Complexity and ultrastructure of infectious extracellular vesicles from cells infected by non-enveloped virus. Sci. Rep. 10, 7939. doi:10.1038/s41598-02064531-1

Yaron, J. R., Gangaraju, S., Rao, M. Y., Kong, X., Zhang, L., Su, F., et al. (2015). $\mathrm{K}(+)$ regulates $\mathrm{Ca}\left(2^{+}\right)$to drive inflammasome signaling: dynamic visualization of ion flux in live cells. Cell Death Dis. 6, e1954. doi:10.1038/ cddis. 2015.277

Yuana, Y., Koning, R. I., Kuil, M. E., Rensen, P. C., Koster, A. J., Bertina, R. M., et al. (2013). Cryo-electron microscopy of extracellular vesicles in fresh plasma. J. Extracell. Vesicles 2. doi:10.3402/jev.v2i0.21494

Zhang, X., Hubal, M. J., and Kraus, V. B. (2020). Immune cell extracellular vesicles and their mitochondrial content decline with ageing. Immun. Ageing 17, 1. doi:10.1186/s12979-019-0172-9

Conflict of Interest: The authors declare that the research was conducted in the absence of any commercial or financial relationships that could be construed as a potential conflict of interest.

The handling editor declared a past co-authorship with one of the authors EA.

Copyright (C) 2021 Lombardi, Gabrielli, Adinolfi and Verderio. This is an open-access article distributed under the terms of the Creative Commons Attribution License (CC $B Y)$. The use, distribution or reproduction in other forums is permitted, provided the original author(s) and the copyright owner(s) are credited and that the original publication in this journal is cited, in accordance with accepted academic practice. No use, distribution or reproduction is permitted which does not comply with these terms. 